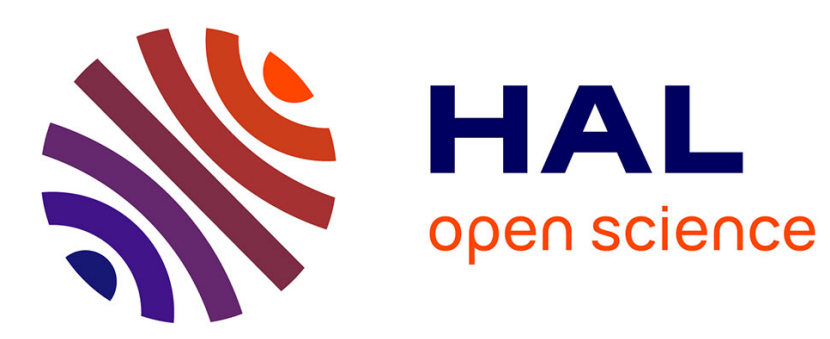

\title{
Consistent vortex initialization for the athermal lattice Boltzmann method
}

Gauthier Wissocq, Jean-François Boussuge, Pierre Sagaut

\section{To cite this version:}

Gauthier Wissocq, Jean-François Boussuge, Pierre Sagaut. Consistent vortex initialization for the athermal lattice Boltzmann method. Physical Review E , 2020, 101 (4), 10.1103/PhysRevE.101.043306 . hal-03229006

\section{HAL Id: hal-03229006 https://hal.science/hal-03229006}

Submitted on 21 May 2021

HAL is a multi-disciplinary open access archive for the deposit and dissemination of scientific research documents, whether they are published or not. The documents may come from teaching and research institutions in France or abroad, or from public or private research centers.
L'archive ouverte pluridisciplinaire HAL, est destinée au dépôt et à la diffusion de documents scientifiques de niveau recherche, publiés ou non, émanant des établissements d'enseignement et de recherche français ou étrangers, des laboratoires publics ou privés. 


\title{
Consistent vortex initialization for the athermal lattice Boltzmann method
}

\author{
Gauthier Wissocq $\odot,{ }^{1,2, *}$ Jean-François Boussuge $\odot,{ }^{1}$ and Pierre Sagaut $\oplus^{3}$ \\ ${ }^{1}$ CERFACS, 42 Avenue G. Coriolis, 31057 Toulouse cedex, France \\ ${ }^{2}$ Safran Aircraft Engines, 77550 Moissy-Cramayel, France \\ ${ }^{3}$ Aix Marseille Univ, CNRS, Centrale Marseille, M2P2 UMR 7340, 13451 Marseille, France
}

\begin{abstract}
A barotropic counterpart of the well-known convected vortex test case is rigorously derived from the Euler equations along with an athermal equation of state. Starting from a given velocity distribution corresponding to an intended flow recirculation, the athermal counterpart of the Euler equations are solved to obtain a consistent density field. The present initialization is assessed on a standard lattice Boltzmann solver based on the D2Q9 lattice. Compared to the usual isentropic initialization, a much lower spurious relaxation toward the targeted solution is observed, which is due to the spatial resolution rather than approximated macroscopic quantities. The amplitude of the spurious waves can be further reduced by including an off-equilibrium part in the initial distribution functions.
\end{abstract}

\section{INTRODUCTION}

The lattice Boltzmann (LB) method has recently emerged as a powerful approach for computational fluid dynamics (CFD) [1-3]. During the past decade, its automatic mesh generation allowing us to easily deal with complex geometries [4], together with a low-dissipative scheme [5] and an efficient easily parallelizable algorithm [6], has made it very attractive for aeronautical applications. Yet, the use of LB solvers to simulate compressible flows and flows with large temperature fluctuations is still challenging, for two main reasons: (1) increasing the physical behavior of a LB method requires the use of more and more discrete velocities $[7,8]$, which may hamper computational efficiency, and (2) the LB scheme is subject to strong numerical instabilities, especially when the Mach number and the temperature fluctuations increase [9]. These instabilities are sometimes attributed to mode coupling [10-12], the occurence of negative distribution functions $[13,14]$, the nonexistence of a H-theorem for the LB scheme [15,16], or an insufficient numerical accuracy [17]. Even though some strategies were proposed to tackle these issues, such as multispeed LB [8,18-20], double distribution function [21-23], or hybrid approaches [24-27], numerical instabilities still restrict these methods to the fields of academic research. However, performing an athermal assumption leads to considerable gains in numerical stability, which is usually adopted in the so-called standard lattice Boltzmann methods. It basically consists in assuming the temperature field of the Maxwell-Boltzmann equilibrium distribution function as constant in space and time. As a consequence, this standard LBM is inherently restricted to simulations of isothermal and weakly compressible flows.

\footnotetext{
*gauthier.wissocq@cerfacs.fr
}

The standard athermal LB method (LBM) is nowadays widely popularized for industrial purpose, e.g., for computational aeroacoustics [28,29] or in the automotive industry [30]. However, it is still subject to many research topics. First, the numerical robustness of the common Bhatnagar-Gross-Krook (BGK) [31] collision model in the zero-viscosity limit (for high Reynolds number flows) is still delicate. Many more sophisticated collision models have been designed to extend their stability range, among which multiple relaxation time (MRT) models [10,32,33], two-relaxation-time (TRT) models [34], the regularized [19,20,35-37], cascaded [38], cumulant $[39,40]$, entropic $[16,41-43]$ models, etc. Understanding the origins of the numerical instabilities, as well as the numerical behavior of these collision models, is an important matter of academic research. Furthermore, local behaviors arising, e.g., at open or closed boundaries $[11,44]$ or at a transition between different mesh resolutions [45], can induce spurious phenomena that may affect the whole simulation domain. These issues are not clearly understood yet and remain within the framework of academic research.

In this context, the simulation of very simple test cases is of paramount importance to assess new LB methods. Periodic cases, such as the double shear layer of Minion and Brown [46] are often preferred in the validation of collision models, so that spurious effects of boundary conditions can be avoided. A reference case for the validation of CFD solvers is the convected vortex test case, which is usually initialized as the analytical Lamb-Oseen vortex [47-49], demonstrated from the isentropic Euler equations. The simplicity of this case has made it very appealing for the assessment of numerical schemes in the fluid core since it can reveal its dissipation, dispersion and isotropy properties [50-52]. Moreover, the convection of a vortex towards a (nonreflecting) boundary [11] or a transition between different mesh resolutions [45] can highlight the aforementioned spurious phenomena in a reduced simulation. 
Unfortunately, in the athermal assumption of the standard LBM, concepts of energy and entropy are meaningless from a physical viewpoint. Instead of obeying the standard ideal gas law ( $p=\rho R T$, where $p, \rho, R$, and $T$, respectively, refer to pressure, density, heat gas constant, and temperature), an athermal fluid flow obeys a so-called barotropic equation of state ( $p=\rho R T_{0}$, where $T_{0}$ is a constant) [53]. Hence, the usual isentropic vortex test case is not consistent with the athermal equations solved by the LBM. The inconsistency between the flow initialization and the macroscopic equations solved by the LB method is known to induce a physical transient adaptation at the origin of spurious waves that may corrupt the study of the phenomena of interest [11,45]. Because of these difficulties, the convected vortex is not usually assessed with the standard LB method, while it is very common for NS based solvers. Gendre et al. [45] have partially got rid of spurious phenomena with a so-called pseudo-isentropic vortex, obtained thanks to a Taylor expansion of the isentropic one for low temperature variations. But there is still a dependency of the heat capacity ratio $\gamma$, which has no meaning in an athermal solver. The aim of this article is to provide a proper initialization of the convected vortex test case in agreement with the barotropic equation of state of the standard LBM and to investigate its dependency on Mach number and spatial resolution. For this reason, it will be referred to as the barotropic vortex test case.

Section II is dedicated to a brief review of the origins of the standard athermal assumption and its consequences on the fluid behavior. In Sec. III, starting from a given velocity field corresponding to an intended recirculation, the Euler equations will be written in cylindrical coordinates and thermodynamic fields in agreement with the equation of state will be obtained for the (1) isentropic and (2) barotropic cases. Relations between the derived density fields will be drawn. In Sec. IV, a numerical validation of the barotropic density field is performed on the D2Q9 lattice for stationary and convected vortices. It is compared with the isentropic one and other initializations that can be found in the literature [45]. A particular attention will be paid to the way distribution functions are initialized.

\section{DISCRETE VELOCITY BOLTZMANN EQUATION AND ATHERMAL APPROXIMATION}

\section{A. Discrete velocity Boltzmann equation}

The LB scheme relies on a particular time and space discretization of the so-called discrete velocity Boltzmann equation (DVBE):

$$
\forall i \in \llbracket 1, V \rrbracket, \quad \frac{\partial f_{i}}{\partial t}+\xi_{i} \cdot \nabla f_{i}=-\frac{1}{\tau}\left(f_{i}-f_{i}^{\mathrm{eq}}\right),
$$

where the Bhatnaghar-Gross-Krook (BGK) [31] approximation has been assumed to model the right-hand-side collision term. In Eq. (1), $\left(f_{i}\right)_{i \in \llbracket 1, V \rrbracket}$ is a set of discrete distribution functions associated to the lattice velocities $\left(\xi_{i}\right)_{i \in \llbracket 1, V \rrbracket}, \tau$ is the relaxation time of the BGK collision model and $\left(f_{i}^{\mathrm{eq}}\right)_{i \in \llbracket 1, V \rrbracket}$ is a set of discrete equilibrium distribution functions, yet to be defined. Note that bold notations stand for vectors and tensors, the central dot denotes the scalar product and $\nabla$ is the gradient operator. Examples of standard lattices of references are provided in the Appendix. Macroscopic quantities of interest such as the mass density $\rho$, fluid velocity $\boldsymbol{u}$, internal energy $e$ and temperature $T$ can be defined as discrete moments of the distribution function:

$$
\begin{gathered}
\rho=\sum_{i=1}^{V} f_{i}, \quad \rho \boldsymbol{u}=\sum_{i=1}^{V} \xi_{i} f_{i}, \\
\rho e=\rho c_{v} T=\sum_{i=1}^{V} \frac{\left\|\xi_{\boldsymbol{i}}-\boldsymbol{u}\right\|^{2}}{2} f_{i},
\end{gathered}
$$

where $c_{v}=(D / 2) R$ is the heat capacity ratio at constant volume of a monatomic gas, $D$ is the spatial dimension and $R$ is the heat gas constant.

A macroscopic interpretation of the DVBE, thanks to, e.g., a Chapman-Enskog expansion [54], allows showing that the underlying physics is completely driven by the statistical moments of the equilibrium distribution functions $\boldsymbol{m}_{\mathrm{eq}}^{(n)}$, defined for a discrete velocity space as

$$
\boldsymbol{m}_{\mathrm{eq}}^{(n)}=\sum_{i=1}^{V} \xi_{i}^{n} f_{i}^{\mathrm{eq}}
$$

where $\xi_{i}{ }^{n}$ denotes the $n$ th-rank tensor built by $n$ tensor products of $\xi_{i}$. A general way to build a set of $\left(f_{i}^{\text {eq }}\right)_{i \in \llbracket 1, V \rrbracket}$ satisfying intended equilibrium moments relies on a Gauss-Hermite quadrature, together with a Hermite polynomial expansion of the equilibrium distribution function [7,18]:

$$
f_{i}^{\mathrm{eq}}=w_{i} \sum_{n=0}^{N} \frac{1}{n ! c_{s}^{2 n}} \boldsymbol{a}_{\mathrm{eq}}^{(n)}: \mathcal{H}_{i}^{(n)} .
$$

In the above equation, ":" stands for the full contraction of indices of two $n$ th-order tensors, $\mathcal{H}_{i}^{(n)}=\mathcal{H}^{(n)}\left(\xi_{i}\right)$ where $\mathcal{H}^{(n)}$ is the $n$ th-order Hermite polynomial defined as

$$
\mathcal{H}^{(n)}(\boldsymbol{\xi})=\frac{\left(-c_{s}^{2}\right)^{n}}{w(\xi)} \frac{\partial^{n} w}{\partial \xi^{n}}, \quad w(\xi)=\frac{1}{\left(2 \pi c_{s}^{2}\right)^{D / 2}} \exp \left(\frac{-\xi^{2}}{2 c_{s}^{2}}\right)
$$

$c_{s}$ is the Newtonian sound speed defined as $c_{s}=\sqrt{R T_{0}}, T_{0}$ is a reference temperature, $\left(w_{i}\right)_{i \in \llbracket 1, V \rrbracket}$ are the weights of the Gauss-Hermite quadrature and $\boldsymbol{a}_{\mathrm{eq}}^{(n)}$ are the so-called Hermite moments of the equilibrium distributions. They are usually imposed to match their continuous counterpart:

$$
\boldsymbol{a}_{\mathrm{eq}}^{(n)}=\int \mathcal{H}^{(n)}(\boldsymbol{\xi}) f^{\mathrm{eq}}(\boldsymbol{\xi}) d \boldsymbol{\xi},
$$

where $f^{\text {eq }}$ is the Maxwell-Boltzmann distribution function [55] (which should not be confused with $\left.\left(f_{i}^{\mathrm{eq}}\right)_{i \in \llbracket 1, V \rrbracket}\right)$ :

$$
f^{\mathrm{eq}}=\frac{\rho}{(2 \pi R T)^{D / 2}} \exp \left(-\frac{\|\boldsymbol{\xi}-\boldsymbol{u}\|^{2}}{2 R T}\right) .
$$

Finally, in Eq. (5), $N$ stands for the higher-order Hermite equilibrium moment that can be recovered with such a polynomial expansion. It should obey $2 N \leqslant Q$, where $Q$ is the order of quadrature of the lattice (cf. the Appendix). 


\section{B. Standard athermal LBM}

The most common lattices introduced in the Appendix have an order of quadrature $Q=5$, meaning that the Hermite polynomial expansion of Eq. (5) cannot theoretically exceed $N=2$. Only equilibrium moments up to the second order can therefore be prescribed. Because of this limitation, macroscopic errors arise at the Navier-Stokes level. They can be attributed, e.g., thanks to a Chapman-Enskog expansion, to discrepancies with the continuous moments of the MaxwellBoltzmann equilibrium Eq. (8):

(1) $\boldsymbol{a}_{\mathrm{eq}}^{(3)}=0$ yields a cubic error in Mach number in the momentum equation,

(2) incorrect third- and fourth-order equilibrium moments yield errors in the transport and diffusive terms of the energy equation.

More details on these error terms can be found in Ref. [20]. The large errors arising in the energy equation are usually circumvented by imposing an athermal flow, i.e., $T=T_{0}$ in the expression of the Maxwell-Boltzmann distribution function Eq. (8). With this assumption, the macroscopic equations obtained at the Navier-Stokes level are

$$
\begin{gathered}
\frac{\partial \rho}{\partial t}+\nabla \cdot(\rho \boldsymbol{u})=0 \\
\frac{\partial(\rho \boldsymbol{u})}{\partial t}+\nabla \cdot(\rho \boldsymbol{u} \boldsymbol{u})+\frac{\partial\left(\rho c_{s}^{2}\right)}{\partial \boldsymbol{x}}=2 \mu \nabla \cdot \boldsymbol{S}+O\left(\boldsymbol{u}^{3}\right)
\end{gathered}
$$

where " $\nabla . "$ stands for the divergence operator, $\mu=\tau \rho c_{s}^{2}$ is the dynamic viscosity, and $\boldsymbol{S}=\left[\boldsymbol{\nabla u}+(\nabla \boldsymbol{u})^{T}\right] / 2$ is the strain rate tensor. Note that Eqs. (9) and (10) form a closed set of equations without involving any notion of energy nor temperature. They are usually considered suitable for isothermal and weakly compressible flows. Notwithstanding the $O\left(\boldsymbol{u}^{3}\right)$ error which is due to an incorrect third-order equilibrium moment, the mass and momentum equations differ from the well-known form of compressible Navier-Stokes equations in several ways. First, a pressure gradient can be identified in Eq. (10), where pressure $p$ is defined as

$$
p=\rho c_{s}^{2}=\rho R T_{0}
$$

It differs from the usual ideal gas equation of state $(p=\rho R T)$ by the fact that $T_{0}$ does not obey any evolution equation, it is rather kept constant. Equation (11) is sometimes referred to as a barotropic equation of state, rather than an ideal gas one [53]. An important consequence is that the speed of sound is equal to the Newtonian sound speed $c_{s}=\sqrt{R T_{0}}$ which is the sound speed at constant temperature rather than at constant entropy. In particular, it does not account for temperature variations nor heat capacity ratio $\gamma$, which have no meaning with the athermal flow assumption. In addition, a shear stress can be identified in Eq. (10) as

$$
\boldsymbol{\sigma}=2 \mu \boldsymbol{S}
$$

Contrary to its Navier-Stokes counterpart, this shear stress is not traceless: it contains a normal stress which is not held by the thermodynamic pressure. This error is usually referred to as a compressibility error [56]. It can be viewed as an add of bulk viscosity $\mu_{b}[56]$, since

$$
\boldsymbol{\sigma}=\mu\left(2 \boldsymbol{S}-\frac{2}{D} \boldsymbol{\nabla} \cdot \boldsymbol{u}\right)+\mu_{b}(\boldsymbol{\nabla} \cdot \boldsymbol{u}) \boldsymbol{I},
$$

with $\mu_{b}=2 \mu / D$.

The LB methods for which a fifth-order quadrature imposes a second-order expansion of the equilibrium distribution function are usually referred to as standard LB methods. Note that, regarding the D2Q9 and D3Q27 lattices, the discrete equilibrium can be improved by including higher-order Hermite polynomials that are second-order per direction [36], i.e.,

$$
\begin{array}{rr}
\text { D2Q9 lattice : } & \mathcal{H}_{x x y}^{(3)}, \mathcal{H}_{x y y}^{(3)}, \mathcal{H}_{x x y y}^{(4)}, \\
\text { D3Q27 lattice : } & \mathcal{H}_{x x y}^{(3)}, \mathcal{H}_{x x z}^{(3)}, \mathcal{H}_{x y y}^{(3)}, \mathcal{H}_{x y z}^{(3)}, \mathcal{H}_{x z z}^{(3)}, \\
& \mathcal{H}_{y y z}^{(3)}, \mathcal{H}_{y z z}^{(3)}, \mathcal{H}_{x x y y}^{(4)}, \mathcal{H}_{x x y z}^{(4)}, \mathcal{H}_{x x z z}^{(4)}, \\
\mathcal{H}_{x y y z}^{(4)}, \mathcal{H}_{x y z z}^{(4)}, \mathcal{H}_{y y z z}^{(4)}, \mathcal{H}_{x x y y z}^{(5)}, \\
\mathcal{H}_{x y y z z}^{(5)}, \mathcal{H}_{x x y y y}^{(5)}, \mathcal{H}_{x x y y z}^{(6)} .
\end{array}
$$

However, even if it may reduce error terms in some specific directions [12], these models are still restricted to isothermal weakly compressible flows.

To circumvent the limitation to athermal flows, several strategies can be adopted:

(1) lattices with a higher order of quadrature can be used, so that the energy equation can be solved without error $[7,8,18]$,

(2) a second set of distribution functions can be introduced so that energy or temperature can be defined as its zeroth-order moment [21-23],

(3) a hybrid approach can be adopted, where the energy equation is solved by finite-differences [25,27].

However, either these solutions do not actually introduce an ideal gas coupling (but, e.g., a Boussinesq coupling), or they lead to severe numerical instabilities after time $\&$ space discretizations [9]. For these reasons, the development of stable thermal LB models is still a topic of research, and the standard athermal LB method is more mature to handle many industrial applications.

The isentropic convected vortex test case, which is one of the academic reference test cases to assess the behavior of Navier-Stokes-based solvers, is not consistent with the athermal assumption done in standard LB methods, where the notion of entropy has no meaning. The purpose of the next section is to provide convected test cases in agreement with both the barotropic equation of state Eq. (11) and the ideal gas one.

\section{DERIVATION OF THE CONVECTED VORTEX}

Let us start writing the (initial) velocity field of an intended convected vortex. In two-dimensional Cartesian coordinates, it reads as

$$
\begin{gathered}
u_{x}=U_{0}-\epsilon\left(\frac{y-y_{c}}{R_{c}}\right) \exp \left[-\frac{\left(x-x_{c}\right)^{2}+\left(y-y_{c}\right)^{2}}{2 R_{c}^{2}}\right], \\
u_{y}=\epsilon\left(\frac{x-x_{c}}{R_{c}}\right) \exp \left[-\frac{\left(x-x_{c}\right)^{2}+\left(y-y_{c}\right)^{2}}{2 R_{c}^{2}}\right],
\end{gathered}
$$


where $U_{0}$ is a constant advection velocity, $\epsilon$ is the (constant) strength of the vortex, $R_{c}$ is the characteristic radius and $\left(x_{c}, y_{c}\right)$ is the position of its center. The velocity field can eventually be re-written in cylindrical coordinates in the moving frame of the vortex as

$$
\boldsymbol{u}=\epsilon \frac{r}{R_{c}} \exp \left(-\frac{r^{2}}{2 R_{c}^{2}}\right) \boldsymbol{e}_{\boldsymbol{\theta}},
$$

where $r$ is the radius starting from the center of the vortex and $\boldsymbol{e}_{\boldsymbol{\theta}}$ is the unitary azimuthal vector. The purpose is now to derive the thermodynamic variables ( $\rho$ only in the athermal case) corresponding to this velocity field so that the initialization can be in agreement with the equations solved by the standard LBM. To this extent, as for the derivation of the isentropic vortex, the Euler equations are used. Moreover, for an inviscid fluid in the co-moving frame, a steady flow is assumed.

In cylindrical coordinates, by denoting, respectively, $u_{r}$ and $u_{\theta}$ the radial and azimuthal components of $\boldsymbol{u}$, the Euler equations for a steady-state flow can be written as

$$
\begin{gathered}
\nabla \cdot(\rho \boldsymbol{u})=0, \\
u_{r} \frac{\partial u_{r}}{\partial r}+\frac{u_{\theta}}{r} \frac{\partial u_{r}}{\partial \theta}-\frac{u_{\theta}^{2}}{r}=-\frac{1}{\rho} \frac{\partial p}{\partial r}, \\
u_{r} \frac{\partial u_{\theta}}{\partial r}+\frac{u_{\theta}}{r} \frac{\partial u_{\theta}}{\partial \theta}+\frac{u_{r} u_{\theta}}{r}=-\frac{1}{\rho r} \frac{\partial p}{\partial \theta} .
\end{gathered}
$$

Using the mass conservation Eq. (17), and knowing that the velocity field Eq. (16) has a null divergence, one obtains

$$
\boldsymbol{u} \cdot \nabla \rho=0 .
$$

Knowing then that the velocity field has an azimuthal component only, the above equation leads to

$$
\frac{\partial \rho}{\partial \theta}=0 \Rightarrow \rho=\rho(r) .
$$

Moreover, injecting the velocity field Eq. (16) into Eq. (19) leads to

$$
\frac{\partial p}{\partial \theta}=0 \Rightarrow p=p(r) .
$$

Finally, injecting Eq. (16) into Eq. (18) yields

$$
\frac{1}{\rho} \frac{d p}{d r}=\frac{u_{\theta}^{2}}{r},
$$

which is the main equation that has to be resolved. In the following, this equation will be solved for two cases:

(1) for the standard compressible Euler equations with the ideal gas law, the standard isentropic vortex will be recovered,

(2) for the athermal form of the Euler equations, a socalled barotropic vortex that may be used as initialization for the standard LBM will be obtained.

\section{A. Solution for the compressible Euler equations: Isentropic vortex}

First, in the compressible case, three thermodynamic variables are unknown: the pressure $p$, density $\rho$, and temperature $T$. Three equations are then required. A first equation is given by Eq. (23). The second equation is provided by the ideal gas equation of state:

$$
p=\rho R T \text {. }
$$

The third equation linking the thermodynamic variables is the Laplace law for an isentropic flow:

$$
\frac{p}{\rho^{\gamma}}=\frac{p_{0}}{\rho_{0}^{\gamma}},
$$

where $\gamma$ is the heat capacity ratio $[\gamma=(D+2) / D$ for a monatomic gas]. $T_{0}, p_{0}$, and $\rho_{0}$, the reference thermodynamic variables, can be chosen as uniform reference values far away from the vortex. Manipulating Eq. (24) with Eq. (25) leads to

$$
\frac{1}{\rho} \frac{d p}{d r}=c_{p} \frac{d T}{d r}
$$

where $c_{p}=\gamma R /(\gamma-1)$. Hence, Eq. (23) becomes

$$
\frac{d T}{d r}=\frac{u_{\theta}^{2}}{r c_{p}}=\frac{\epsilon^{2}}{c_{p}} \frac{r}{R_{c}^{2}} \exp \left(-\frac{r^{2}}{R_{c}^{2}}\right) .
$$

The integration of this equation is straightforward and gives

$$
T(r)=T_{0}-\frac{1}{2} \frac{\epsilon^{2}}{c_{p}} \exp \left(-\frac{r^{2}}{R_{c}^{2}}\right) .
$$

The density field can then be computed thanks to the Laplace law:

$$
\rho=\rho_{0}\left[1-\frac{(\gamma-1)}{\gamma} \frac{\epsilon^{2}}{2 c_{s}^{2}} \exp \left(-\frac{r^{2}}{R_{c}^{2}}\right)\right]^{1 /(\gamma-1)},
$$

and then the pressure thanks to the ideal gas equation of state. This solution corresponds to the usual isentropic Lamb-Oseen $[47,49]$ vortex.

\section{B. A barotropic vortex for the standard athermal LBM}

As discussed in Sec. II, in the standard lattice Boltzmann solvers, an athermal form of the Navier-Stokes equations is obtained. Pressure and density fields are linked with each other through the barotropic equation of state Eq. (11) instead of the ideal gas equation of state Eq. (24). Directly inserting Eq. (11) into Eq. (23) leads to

$$
\frac{1}{\rho} \frac{d \rho}{d r}=\frac{u_{\theta}^{2}}{r c_{s}^{2}} .
$$

This single equation is sufficient to obtain the density field. It can be rewritten as

$$
\frac{d(\ln \rho)}{d r}=\left(\frac{\epsilon}{c_{s}}\right)^{2} \frac{r}{R_{c}^{2}} \exp \left(-\frac{r^{2}}{R_{c}^{2}}\right) .
$$

The integration of this equation is straightforward and gives

$$
\rho(r)=\rho_{0} \exp \left[-\frac{\epsilon^{2}}{2 c_{s}^{2}} \exp \left(-\frac{r^{2}}{R_{c}^{2}}\right)\right],
$$

which is the expression that will be further referred to as the density field for a barotropic vortex.

Note that this expression of the density field can be approximated thanks to a Taylor expansion if $\epsilon / c_{s} \ll 1$. At the second order in $\epsilon / c_{s}$, it leads to the following vortex:

$$
\rho(r)=\rho_{0}\left[1-\frac{\epsilon^{2}}{2 c_{s}^{2}} \exp \left(-\frac{r^{2}}{R_{c}^{2}}\right)\right]+O\left(\frac{\epsilon^{4}}{c_{s}^{4}}\right) .
$$


This expression corresponds to the so-called pseudoisentropic vortex used in Ref. [45] for the numerical assessment of a vortex convected across a mesh transition.

\section{Relations between the isentropic and the barotropic vortices}

A direct link can be drawn between the density distributions obtained for the isentropic and the barotropic vortices by noticing that the athermal flow assumption is equivalent to the ideal gas modeling in the limit of adiabatic exponent $\gamma=1$. This is in disagreement with a monatomic gas modeling, for which $\gamma=(D+2) / D$. Indeed, in the compressible case, the internal energy conservation of the Navier-Stokes equations reads

$$
\frac{\partial(\rho e)}{\partial t}+\nabla \cdot(\rho e \boldsymbol{u})=-p \nabla \cdot \boldsymbol{u}+\nabla \cdot \boldsymbol{q}+\boldsymbol{\sigma}: \boldsymbol{u},
$$

where $\boldsymbol{q}$ is the heat flux. Noticing that $\rho e=\rho c_{v} T=p /(\gamma-$ 1 ), this equation can equivalently be rewritten as

$$
\frac{\partial p}{\partial t}+\nabla \cdot(p \boldsymbol{u})=(1-\gamma)(p \nabla \cdot \boldsymbol{u}+\nabla \cdot \boldsymbol{q}+\boldsymbol{\sigma}: \boldsymbol{u}) .
$$

In the particular case $\gamma=1$, compressibility effects driven by the $p \boldsymbol{\nabla} \cdot \boldsymbol{u}$ term of the above equation have no influence on the evolution of pressure, as well as heat conduction and viscous effects. Pressure rather obeys a simple transport equation, exactly like $\rho$ in the mass conservation Eq. (9). Hence, if pressure is initialized as proportional to the density field and since they both obey the same evolution equation, then $p$ will be nothing more than the density by a constant factor. Using the ideal gas law, this constant factor can be identified as $R T_{0}$, so that the barotropic equation of state of Eq. (11) is obtained. The exact behavior of the athermal assumption of Sec. II is therefore recovered. In this limit, the isentropic sound speed $(\sqrt{\gamma R T})$ is reduced to the Newtonian one $\left(c_{s}=\sqrt{R T_{0}}\right)$.

Let us now compute the density distribution of the isentropic vortex in the limit $\gamma \rightarrow 1$. To this extent, one can compute the $\log$ of Eq. (29) and perform an asymptotic expansion about $\gamma=1$ :

$$
\begin{aligned}
\log \left(\rho / \rho_{0}\right) & =\frac{1}{\gamma-1} \log \left[1-\frac{(\gamma-1)}{2} \frac{\epsilon^{2}}{2 c_{s}^{2}} \exp \left(-\frac{r^{2}}{R_{c}^{2}}\right)\right] \\
& =\frac{1}{\gamma-1}\left[-\frac{(\gamma-1)}{\gamma} \exp \left(-\frac{r^{2}}{R_{c}^{2}}\right)+O\left((\gamma-1)^{2}\right)\right] \\
& \underset{\gamma \rightarrow 1}{\longrightarrow}-\exp \left(-\frac{r^{2}}{R_{c}^{2}}\right),
\end{aligned}
$$

so that

$$
\rho \underset{\gamma \rightarrow 1}{\longrightarrow} \rho_{0} \exp \left[-\frac{\epsilon^{2}}{2 c_{s}^{2}} \exp \left(-\frac{r^{2}}{R_{c}^{2}}\right)\right] .
$$

The density distribution of Eq. (32) is recovered, so that the barotropic vortex is the limit of the isentropic one in the limit $\gamma \rightarrow 1$.

\section{NUMERICAL VALIDATION}

Let us now assess the consistency of the barotropic density field by setting it as the initial solution of a standard athermal LB solver. In the following, considering the initial velocity field given in Eqs. (14) and (15), four initial density fields are studied:

(1) the "standard" isentropic vortex of Eq. (29),

(2) the first-order Taylor expansion of Ref. [45], cf. Eq. (33),

(3) a second-order Taylor expansion of Ref. [45]:

$$
\rho=\rho_{0}\left[1-\frac{\epsilon^{2}}{2 c_{s}^{2}} \exp \left(-\frac{r^{2}}{R_{c}^{2}}\right)+\frac{\epsilon^{4}}{8 \gamma c_{s}^{4}} \exp \left(-\frac{2 r^{2}}{R_{c}^{2}}\right)\right],
$$

(4) the barotropic vortex of Eq. (32).

To impose initial distribution functions from known macroscopic conditions, two strategies will be adopted in the following. The first one consists in initializing the distribution functions to their equilibrium value $f_{i}^{\text {eq }}$. In the second-one, an off-equilibrium part is included by computing the velocity gradients, as proposed, e.g., in Refs. [57,58]. It can be computed thanks to the first-order term of the Chapman-Enskog expansion, truncated up to its second-order term in Hermite polynomials:

$$
f_{i}=f_{i}^{\mathrm{eq}}+f_{i}^{(1)}, \quad f_{i}^{(1)}=\frac{w_{i}}{2 c_{s}^{4}} \boldsymbol{a}_{1}^{\mathrm{neq}}: \mathcal{H}_{i}^{(2)},
$$

with

$$
\boldsymbol{a}_{1}^{\mathrm{neq}}=\boldsymbol{\sigma}=2 \rho \tau c_{s}^{2} \boldsymbol{S}, \quad \mathcal{H}_{i, \alpha \beta}^{(2)}=\xi_{\alpha} \xi_{\beta}-c_{s}^{2} \delta_{\alpha \beta},
$$

where the velocity gradients in the strain rate tensor $S$ can be computed analytically.

Two cases will be investigated:

(1) a static vortex, to highlight an eventual adaptation in the form of acoustic waves and decrease in the vortex amplitude,

(2) a convected vortex, to possibly highlight the effects of nonhydrodynamic modes that would not be convected in the static case.

All the cases are performed with the D2Q9 lattice [2] and a BGK collision model. In the following, $f_{i}^{\text {eq }}$ is chosen as a "complete" expansion of the Maxwell-Boltzmann equilibrium distribution function onto the Hermite polynomial basis of the D2Q9 lattice of nine elements [18,20,36]:

$$
\begin{aligned}
f_{i}^{\mathrm{eq}}= & w_{i} \rho\left[1+\frac{\boldsymbol{\xi}_{\boldsymbol{i}} \cdot \boldsymbol{u}}{c_{s}^{2}}+\frac{\left(\boldsymbol{\xi}_{\boldsymbol{i}} \cdot \boldsymbol{u}\right)^{2}}{2 c_{s}^{4}}-\frac{\|\boldsymbol{u}\|^{2}}{2 c_{s}^{2}}\right] \\
& +\frac{w_{i}}{2 c_{s}^{6}}\left[\mathcal{H}_{i, x x y}^{(3)} a_{\mathrm{eq}, x x y}^{(3)}+\mathcal{H}_{i, x y y}^{(3)} a_{\mathrm{eq}, x y y}^{(3)}\right] \\
& +\frac{w_{i}}{4 c_{s}^{8}} \mathcal{H}_{i, x x y y}^{(4)} a_{\mathrm{eq}, x x y y}^{(4)},
\end{aligned}
$$

with

$$
\begin{gathered}
a_{\mathrm{eq}, \alpha \beta \gamma}^{(3)}=\rho u_{\alpha} u_{\beta} u_{\gamma}, \quad a_{\mathrm{eq}, x x y y}^{(4)}=\rho u_{x}^{2} u_{y}^{2}, \\
\mathcal{H}_{i, \alpha \beta \gamma}^{(3)}=\xi_{i, \alpha} \xi_{i, \beta} \xi_{i, \gamma}-c_{s}^{2}\left(\delta_{\alpha \beta} \xi_{i, \gamma}+\delta_{\alpha \gamma} \xi_{i, \beta}+\delta_{\beta \gamma} \xi_{i, \alpha}\right), \\
\mathcal{H}_{i, x x y y}^{(4)}=\xi_{i, x}^{2} \xi_{i, y}^{2}-c_{s}^{2}\left(\xi_{i, x}^{2}+\xi_{i, y}^{2}\right)+c_{s}^{4} .
\end{gathered}
$$

This choice is done to increase the robustness of the BGK-LB scheme $[9,36,59]$. 


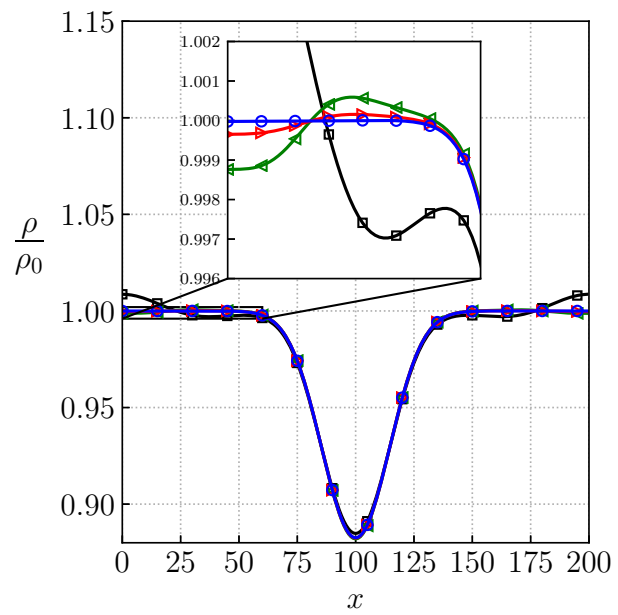

(a) $\epsilon=0.5 c_{s}$

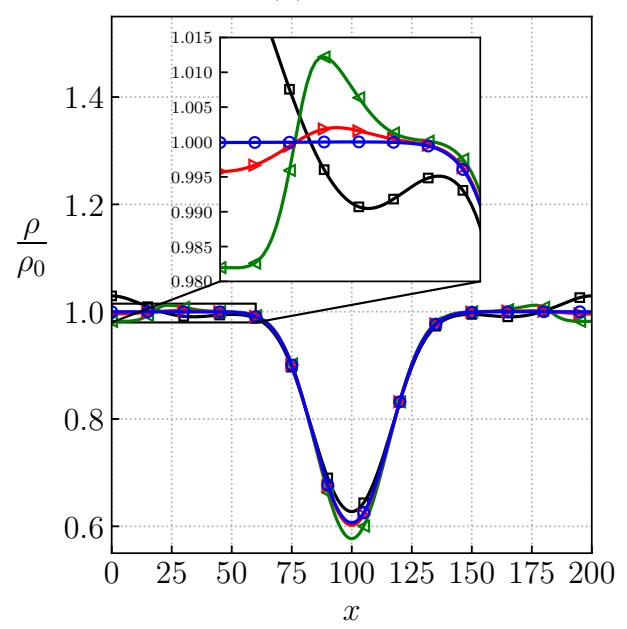

(b) $\epsilon=c_{s}$

FIG. 1. Dimensionless density profile of the static vortex test case after 150 iterations. $\square$ : isentropic vortex; $\triangle$ : first-order approximation of Ref. [45]; $\rightarrow$ : second-order approximation of Ref. [45]; - : barotropic vortex. (a) $\epsilon=0.5 c_{s}$, (b) $\epsilon=c_{s}$.

\section{A. Stationary vortex}

All the parameters of this test case are given below in dimensionless units. A periodic square box of size $(200 \times$ 200) Cartesian points (or voxels) is first considered, with the vortex placed at its center, i.e., $\left(x_{c}, y_{c}\right)=(100,100)$. The characteristic radius is set to $R_{c}=20$ voxels and the reference density is $\rho_{0}=1$. As a naive choice for Eqs. (29) and (38), the heat capacity ratio is intentionally set to $\gamma=1.4$. The relaxation time of the BGK model is set to $\tau=10^{-5}$ to minimize viscous damping and the convective velocity is set to $U_{0}=0$ to simulate a stationary vortex. In all the results presented below, only the results obtained with an initialization at equilibrium will be shown. The effect of adding an off-equilibrium part in the initial distribution functions will be investigated in the next section. Finally, two values for the parameter $\epsilon$ are studied: (1) $\epsilon=0.5 c_{s}$, (2) $\epsilon=c_{s}$. The first case leads to a maximal Mach number of 0.3 , and the second one to 0.6. The choice of the second case can be discussed regarding the coherence of an athermal model with a local Mach number of 0.6 , notwithstanding the stability issues encountered with the BGK model at this Mach number. However, here, only the consistency of the initialization is investigated (rather than whether the behavior is physical or not), and no growing instabilities are observed during the transient time studied below. This second case is only chosen to better highlight the phenomena of interest.

A comparison of the density profiles for the four initializations and the two values of $\epsilon$ after 150 iterations is shown on Fig. 1. An adaptation, which behaves as an acoustic wave starting from the center of the vortex, is observed for the isentropic initialization and for the two kinds of Taylor expansions given in Ref. [45]. As expected, and as noticed by Gendre et al. [45], the adaptation is all the more reduced as the order of the Taylor expansion is increased. Yet, it is still visible on Fig. 1. On the contrary, with the barotropic initialization, this adaptation does not seem to be present any more.

Ratios between the maximal value of $\rho$ (linked to the amplitude of the generated acoustic wave) and the minimal value of $\rho$ (linked to the amplitude of the vortex) are compiled in Table I. It allows quantifying the amplitude of the generated acoustic wave relative to the density fluctuations induced by the vortex. Two meshes are investigated: $(200 \times 200)$ and $(400 \times 400)$ voxels. In the second case, $R_{c}=40$ voxels per characteristic radius.

With the isentropic initialization, the amplitude of the generated acoustic wave is of the same order of magnitude whatever the mesh resolution and relatively large compared to the vortex amplitude (about 10\%). As expected, for both the first- and second-order Taylor expansions, the acoustic adaptation is all the more important as the value of $\epsilon / c_{s}, a$ fortiori the local Mach number, increases. Moreover, like for the isentropic one, the mesh size has no real effect on the adaptation. With the barotropic initialization, two observations can be made. First, the amplitude of the adaptation does not depend on the strength of the vortex $\epsilon / c_{s}$. This means that the accuracy of the barotropic initialization does not depend on the strength of the vortex, contrary to the Taylor expansion proposed in [45]. Secondly, when refining the mesh size from

TABLE I. Ratio $\max \left(\rho-\rho_{0}\right) / \max \left(\rho_{0}-\rho\right)$ characterizing the amplitude of the acoustic wave generated by the initialization relative to the amplitude of the vortex. Maximal and minimal values are computed on the full computational domain between 0 and 150 iterations for $(200 \times 200)$ voxels and between 0 and 300 iterations for $(400 \times 400)$ voxels.

\begin{tabular}{lccccc}
\hline \hline & $\left(N_{x} \times N_{y}\right)$ & Isentropic & Taylor first-order [45] & Taylor second-order [45] & Barotropic \\
\hline$\epsilon=0.5 c_{s}$ & $(200 \times 200)$ & $9.9 \%$ & $0.51 \%$ & $0.14 \%$ & $0.0098 \%$ \\
& $(400 \times 400)$ & $10 \%$ & $0.50 \%$ & $0.13 \%$ & $0.0016 \%$ \\
$\epsilon=c_{s}$ & $(200 \times 200)$ & $9.2 \%$ & $2.9 \%$ & $0.55 \%$ & $0.0093 \%$ \\
& $(400 \times 400)$ & $9.2 \%$ & $2.8 \%$ & $0.54 \%$ & $0.0017 \%$ \\
\hline \hline
\end{tabular}




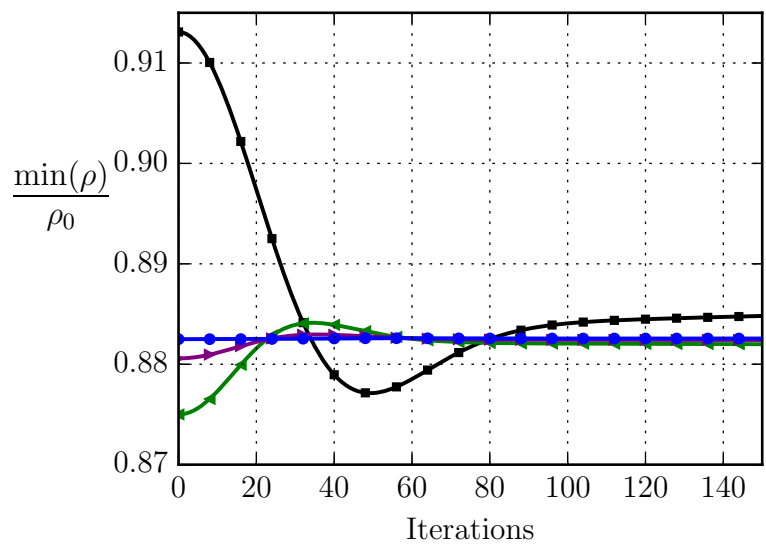

(a) $\epsilon=0.5 c_{s}$

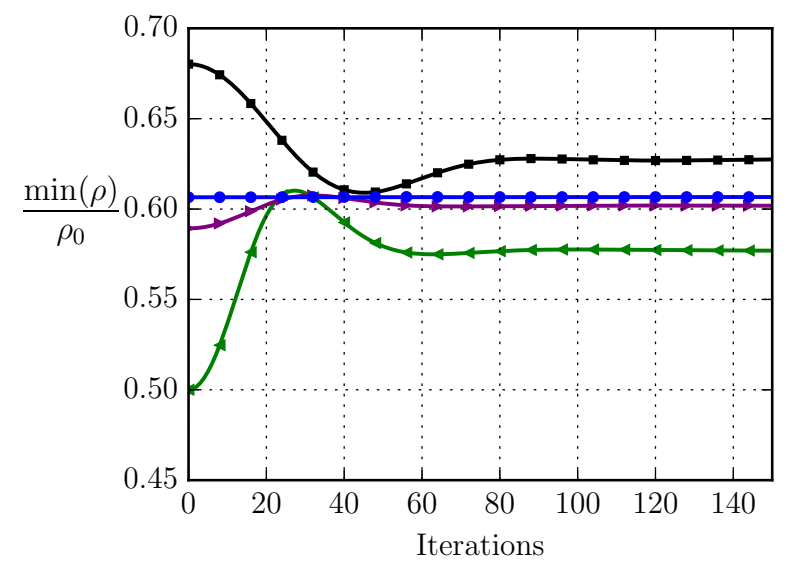

(b) $\epsilon=c_{s}$

FIG. 2. Temporal evolution of the minimal value of the density field, characterizing the evolution of the vortex amplitude in the steady case. $\rightarrow-$ : isentropic vortex; $\multimap$ : first-order approximation of Ref. [45]; $\rightarrow$ : second-order approximation of Ref. [45]; $\rightarrow-$ : barotropic vortex. (a) $\epsilon=0.5 c_{s}$, (b) $\epsilon=c_{s}$.

$(200 \times 200)$ voxels to $(400 \times 400)$ voxels (and $R_{c}$ from 20 voxels to 40 voxels), the amplitude of the acoustic adaptation is divided by six. The impact of the mesh resolution on the density fluctuations is a clear indicator of the consistency of the initial conditions. When initializing the vortex test case with the isentropic solution or its Taylor expansions, spatial discretization errors are largely dominated by the inconsistency of the initial condition. It is then impossible to reduce the acoustic adaptation by refining the mesh resolution. On the contrary, with the barotropic initialization, the fact that this adaptation can be decreased on a finer mesh indicates that the residual adaptation is only due to the spatial discretization, rather than to an incorrect initial state.

Finally, time evolutions of the minimal value of the density field are displayed on Fig. 2 for the two vortices $\left(\epsilon=0.5 c_{s}\right.$ and $\left.\epsilon=c_{s}\right)$ with the $(200 \times 200)$ mesh. It allows highlighting temporal fluctuations of the vortex amplitude. Density fluctuations can be observed after the isentropic initialization, as well as with the two kinds of Taylor expansions. They are linked with the formation of the aforementioned acoustic waves. These oscillations can be problematic since they may be mistaken with a dissipative behavior of the numerical scheme in the first transient steps of a simulation. On the contrary, no oscillations seem to be observed with the barotropic initialization for which the vortex amplitude stay constant.

\section{B. Convected vortex}

Apart from spurious acoustic waves, an inconsistent initialization of the lattice Boltzmann distribution functions may result in the excitation of nonhydrodynamic modes that can be advected in the whole computation domain [53,59-62]. Previous works have highlighted the fact that these modes have a phase velocity close to $\pi$, so that their amplitude is reversed at each iteration [59]. Moreover, in absence of mean flow, they can have a null group velocity [59], which makes them undetectable in the stationary test cases of the previous section. To highlight the effect of the vortex initialization on the generated nonhydrodynamic modes, convected vortex simulations at different Mach numbers $\mathrm{Ma}_{0}=U_{0} / c_{s}$ are carried out in this section. Note that thanks to the Galilean invariance of the Euler equation, the macroscopic initialization of the density field is not affected by the mean flow Mach number.

Test cases similar as in the previous section are performed on a periodic two-dimensional square domain of size $(600 \times$ 600) Cartesian cells, with a convected vortex initially placed at its center, i.e., $\left(x_{c}, y_{c}\right)=(300 \times 300)$. Since the aim of the present work is not to assess the behavior of a particular collision model, the very standard BGK approximation is adopted here, with an equilibrium distribution function expanded up to the fourth-order and $\tau=10^{-5}$. The horizontal convective Mach number is set to $\mathrm{Ma}_{0}=0.3$. For reasons of numerical stability, only the $\epsilon=0.5 c_{s}$ case will be presented below. The characteristic radius of the vortex is set to $R_{c}=5$ voxels. This rather coarse case is adopted to exhibit the nonhydrodynamic behaviors presented below.

Figure 3 displays the horizontal velocity distribution (in lattice units) along the cut $y=300$. The four kinds of density initializations [isentropic Eq. (29), first-order Taylor Eq. (33), second-order Taylor Eq. (38), and barotropic Eq. (32)] are represented, as well as the two kinds of distribution initializations [at equilibrium or with an off-equilibrium part Eq. (39)] at different instants: $t=0, t=350$ and $t=351$ iterations. Several observations are worth noting. First, while $u_{x}-U_{0}=$ 0 along the $(y=300)$-line at $t=0$, it is no more the case after convecting the vortex. It is probably a simple effect of the dispersion properties of the LB scheme, which may shift the central position of the vortex. Moreover, two spurious acoustic waves (denoted by boxes B and C) can be, as previously, identified. As for the stationary vortex case, their amplitude is decreased when adopting the more adapted barotropic vortex. Note that adding an off-equilibrium part also helps decreasing the amplitude of these spurious waves. For instance, with the second-order Taylor initialization of Eq. (38), when initializing $f_{i}^{(1)}$, the amplitudes of the acoustic waves are in the same order of magnitude as with the barotropic initialization Eq. (32). Finally, an upstream convected nonhydrodynamic wave can be observed in all the cases of Fig. 3 (cf. box A). It can be clearly identified thanks to its amplitude inversion between $t=350$ and $t=351$ iterations, which is the evidence of a phase velocity close to $\pi$. This wave is not physical 

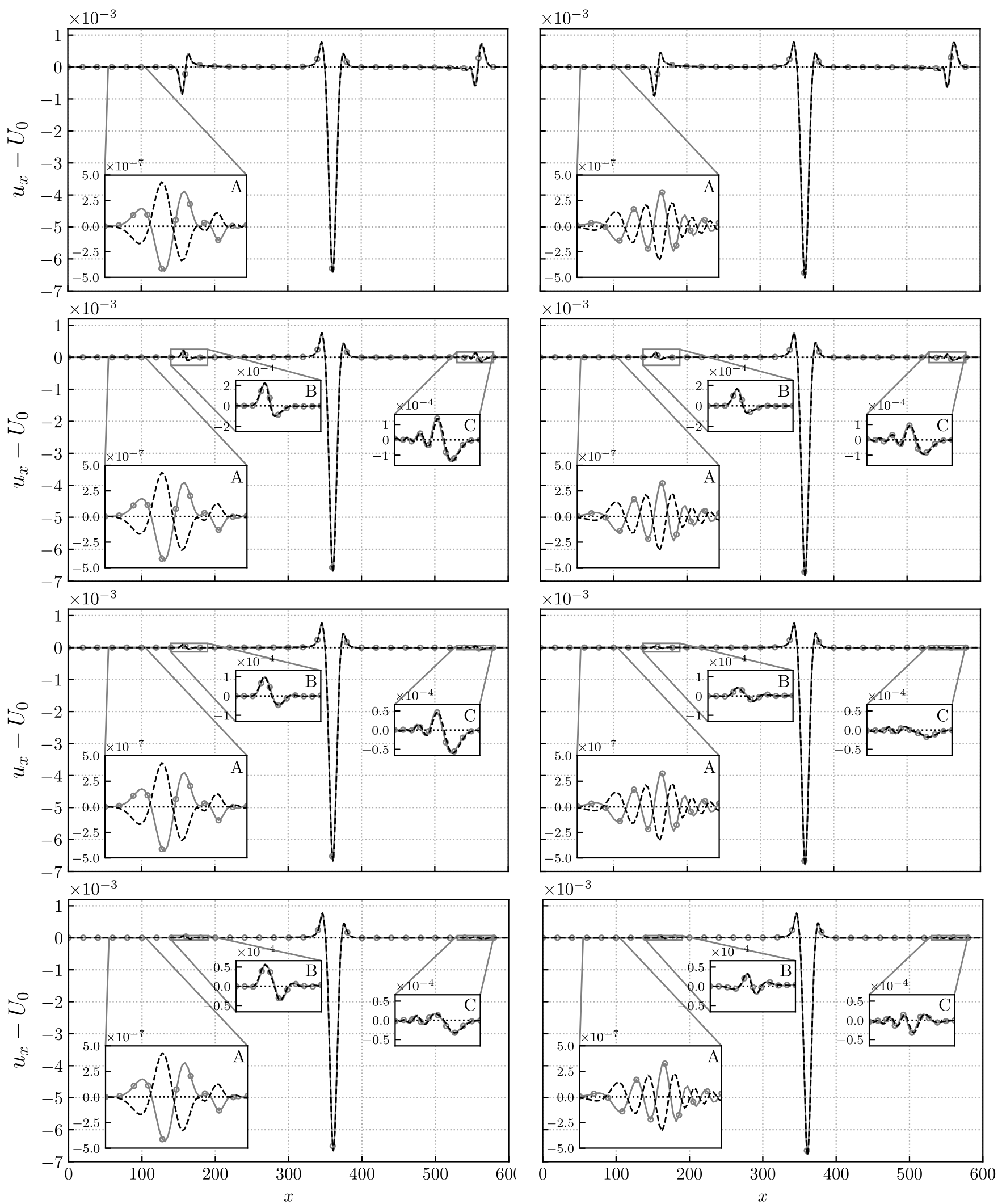

FIG. 3. Horizontal velocity distribution of a vortex convected at $\mathrm{Ma}_{0}=0.3$. From top to bottom: Isentropic Eq. (29), Taylor first-order Eq. (33), Taylor second-order Eq. (38), and barotropic (32) initializations. Left: initialization with equilibrium distribution function, right: with an off-equilibrium part. ....: $t=0 ; \ldots: t=350$; - $-: t=351$ iterations. (A) Spurious nonhydrodynamic wave, (B, C) spurious acoustic waves. 
since it is advected at a higher group velocity than the speed of sound. Moreover, it could not be observed on the static vortex test case, for which its group velocity is probably null, as already stated in Ref. [59]. The shape and the amplitude of this wave is exactly the same whatever the density field initialization. In fact, only the way of initializing the distribution functions seems to impact this wave. Adding an initial off-equilibrium part helps reducing its amplitude, even if only a slight positive effect is observed. Although its amplitude is, in any case, smaller than that of the spurious acoustic waves, the behavior of this kind of nonhydrodynamic wave at a mesh boundary should be treated with care, especially because of its amplitude inversion at each iteration.

To summarize these observations, two effects can be clearly distinguished:

(1) a change in the initial macroscopic fields affects the generation of spurious acoustic waves, but not the nonhydrodynamic ones,

(2) initializing the distribution functions with an offequilibrium parts reduces the amplitude of any spurious wave.

Ratio of the amplitudes of the emitted acoustic wave with respect to that of the vortex $\Delta \rho_{\mathrm{ac}} / \Delta \rho_{\text {covo }}$ [with $\Delta \rho_{\mathrm{ac}}=$ $\max \left(\rho-\rho_{0}\right)$ and $\left.\Delta \rho_{\text {covo }}=\max \left(\rho_{0}-\rho\right)\right]$ are displayed on Fig. 4 for the four initial density fields and the two kinds of distribution initializations. Five vortex resolutions are compared $\left(R_{c}=2.5, R_{c}=5, R_{c}=10, R_{c}=20\right.$, and $\left.R_{c}=40\right)$ for three convective Mach numbers: $\mathrm{Ma}_{0}=0, \mathrm{Ma}=0.3 x$ aligned, and $\mathrm{Ma}_{0}=0.3$ with an angle $\theta_{0}=45^{\circ}$. For each case, the size of the computational domain in terms of number of voxels is 20 times larger than the vortex radius. Moreover, the simulation time is set to $10 R_{c}$ iterations to ensure that spurious waves are well separated from the vortex and to avoid their superposition at the periodic boundaries. In all the computations, the spurious nonhydrodynamic waves do not have a significant amplitude compared to the acoustic ones. Overall, a similar behavior can be noticed whatever the Mach number and the orientation of the mean flow, and the following conclusions can be drawn.

(1) With the isentropic, the first- and second-order Taylor initializations, mesh refinement only has an effect on the spurious adaptation for $R_{c}<5$. With finer spatial resolutions, the inconsistency error dominates, which explains the observation of a plateau in the mesh convergence.

(2) In any case, the effect of initializing the offequilibrium part is similar to a mesh refinement. It can be viewed as an increase in the precision order of the initialization.

(3) With the barotropic initialization, increasing the spatial resolution reduces the amplitude of spurious waves even for the finer cases, and no plateau is observed. Moreover, an asymptotic behavior with a slope -2 is obtained, in agreement with the spatial error of the LB numerical scheme [21,53,63]. This means that the residual adaptation is only due to the vortex resolution, further confirming the consistency of the initialization.

\section{CONCLUSION}

Density distributions of the standard isentropic vortex and the barotropic one have been derived from the Euler equa-

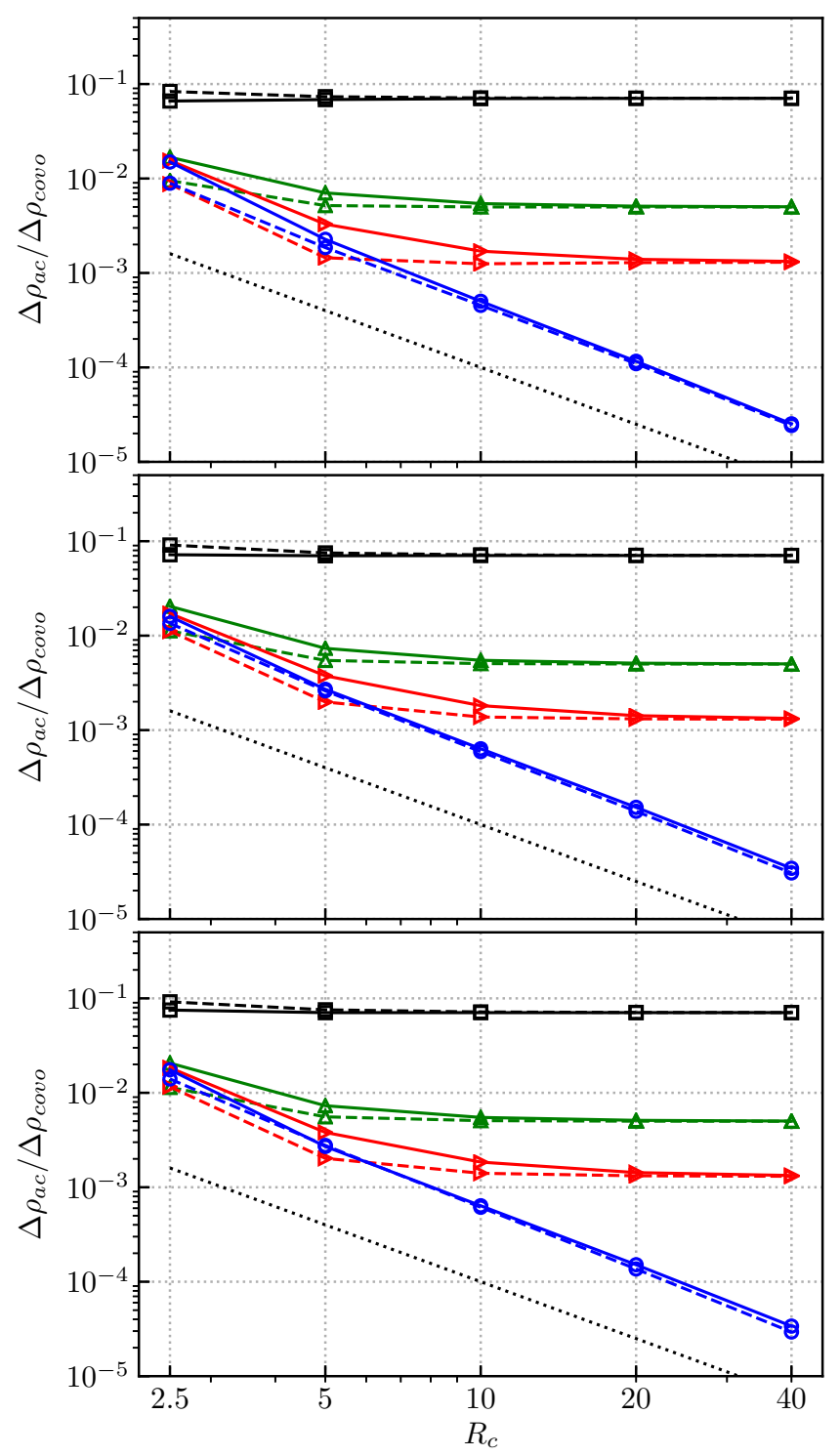

FIG. 4. Ratio of acoustic waves amplitude $\Delta_{\mathrm{ac}}=\max \left(\rho-\rho_{0}\right)$ over the vortex amplitude $\Delta_{\text {covo }}=\max \left(\rho_{0}-\rho\right)$ as function of the vortex resolution $R_{c}$ in terms of number of voxels. Three convective fields are evaluated: (top) $\mathrm{Ma}_{0}=0$, (middle) $x$-aligned flow at $\mathrm{Ma}_{0}=0.3$, (bottom) $\mathrm{Ma}_{0}=0.3$ with incidence $\theta_{0}=45^{\circ}$. $\square$ : isentropic vortex; $\triangle$ : first-order Taylor approximation of Ref. [45]; $\rightarrow$ : second-order approximation of Ref. [45]; ๑-: barotropic vortex. Solid line: initialization at equilibrium; dashed line: initialization of the off-equilibrium part. Dotted line: -2-slope.

tions with the ideal gas equation of state Eq. (24) and the barotropic one Eq. (11), respectively. Relations between both vortices have been established by noticing that the athermal fluid assumption of the standard LBM can be recovered from the compressible equations in the limit of a heat capacity ratio $\gamma \rightarrow 1$. Numerical assessment has been performed on stationary and convected vortices with the aim of highlighting the consistency of the barotropic vortex initialization for the standard athermal LBM. For this reason, it has been compared with other initializations of density field, several mesh resolutions, two values of vortex strengths and two kinds of 
initializations of the LB distribution functions: at equilibrium or with an analytically computed off-equilibrium part. Results highlight the good consistency of the barotropic vortex with the athermal LBM. It minimizes the spurious adaptation that can pollute an academic simulation in the aim of validating a LB model. The remaining acoustic adaptation is independent of the vortex strength, contrary to previous solutions based on a Taylor expansion of the isentropic vortex [45], for which a plateau has been obtained in the mesh convergence. It can be further reduced, either by refining the mesh, or by including the off-equilibrium part in the initial distributions which increases the precision of the initial condition. Moreover, the presence of a nonhydrodynamic wave, advected at a larger velocity than the speed of sound, has been identified, especially when the vortex is convected. Damping the amplitude of this wave has only been possible by a change of initial distribution functions or spatial resolution, rather than their macroscopic value. Even if its amplitude seems much lower than that of the spurious acoustic ones, this wave can be problematic because of its unphysical phase shift at each iteration. Further work may focus on the behavior of this particular wave, e.g., how to effectively reduce its initial contribution or how do more sophistical collision models behave with nonhydrodynamic phenomena.

\section{ACKNOWLEDGMENTS}

The authors acknowledge the financial support of Safran Aircraft Engines and ANRT/CIFRE. Fruitful discussions with
Thomas Astoul have been particularly appreciated. Finally, the authors thank the reviewers for their constructive remarks.

\section{APPENDIX: STANDARD LATTICES}

Two- and three-dimensional lattices commonly used in the so-called standard lattice Boltzmann method are described below. For a sake of clarity, all velocities obtained by cyclic permutations with respect to each Cartesian axis are omitted, the number of velocities belonging to a same group (=same velocity norm) being denoted by $p$. Note that $\boldsymbol{e}_{i}=\xi_{i} \Delta t / \Delta x$ and $c_{s}$ is here the nondimensional Newtonian sound speed: $c_{s}=\sqrt{R T_{0}} \Delta t / \Delta x$.

\begin{tabular}{lcccccc}
\hline \hline Lattice & Quadrature order & $\boldsymbol{e}_{\boldsymbol{i}}$ & Group & $p$ & $w_{i}$ & $c_{s}$ \\
\hline \multirow{3}{*}{ D2Q9 } & \multirow{2}{*}{$Q=5$} & $(1,0)$ & 1 & 1 & $4 / 9$ & \\
& & $(1,1)$ & 3 & 4 & $1 / 36$ & \\
& & $(0,0,0)$ & 1 & 1 & $1 / 3$ & \\
D3Q19 & \multirow{2}{*}{$Q=5$} & $(1,0,0)$ & 2 & 6 & $1 / 18$ & $1 / \sqrt{3}$ \\
& & $(1,1,0)$ & 3 & 12 & $1 / 36$ & \\
& & $(0,0,0)$ & 1 & 1 & $8 / 27$ & \\
D3Q27 & \multirow{2}{*}{$Q=5$} & $(1,0,0)$ & 2 & 6 & $2 / 27$ & $1 / \sqrt{3}$ \\
& & $(1,1,0)$ & 3 & 12 & $1 / 54$ & \\
& & $(1,1,1)$ & 4 & 8 & $1 / 216$ & \\
\hline \hline
\end{tabular}

[1] G. R. McNamara and G. Zanetti, Phys. Rev. Lett. 61, 2332 (1988).

[2] Y. H. Qian, D. D’Humières, and P. Lallemand, Europhys. Lett. 17, 479 (1992).

[3] S. Chen and G. D. Doolen, Annu. Rev. Fluid Mech. 30, 329 (1998).

[4] H. Touil, D. Ricot, and E. Lévêque, J. Comput. Phys. 256, 220 (2014).

[5] S. Marié, D. Ricot, and P. Sagaut, J. Comput. Phys. 228, 1056 (2009).

[6] F. Schornbaum and U. Rüde, SIAM J. Sci. Comput. 38, C96 (2016).

[7] X. Shan, X.-F. Yuan, and H. Chen, J. Fluid Mech. 550, 413 (2006).

[8] X. Shan, J. Comput. Sci. 17, 475 (2016).

[9] D. N. Siebert, L. A. Hegele, and P. C. Philippi, Phys. Rev. E 77, 026707 (2008).

[10] P. Lallemand and L.-S. Luo, Phys. Rev. E 61, 6546 (2000).

[11] G. Wissocq, N. Gourdain, O. Malaspinas, and A. Eyssartier, J. Comput. Phys. 331, 1 (2017).

[12] G. Wissocq, Ph.D. thesis, Aix-Marseille Université, 2019.

[13] Y. Li, R. Shock, R. Zhang, and H. Chen, J. Fluid Mech. 519, 273 (2004).

[14] X. Nie, X. Shan, and H. Chen, in Proceedings of the 47th AIAA Aerospace Sciences Meeting including The New Horizons Forum and Aerospace Exposition, January (American Institute of Aeronautics and Astronautics, Reston, VA, 2009), p. 2009.
[15] I. Karlin, A. Ferrante, and H. C. Öttinger, Europhys. Lett. 47, 182 (1999).

[16] B. M. Boghosian, J. Yepez, P. V. Coveney, and A. Wager, Proc. R. Soc. A 457, 717 (2001).

[17] Y. Li and X. Shan, Philos. Trans. R. Soc., A 369, 2371 (2011).

[18] P. C. Philippi, L. A. Hegele, L. O. E. dos Santos, and R. Surmas, Phys. Rev. E 73, 056702 (2006).

[19] K. K. Mattila, P. C. Philippi, and L. A. Hegele, Phys. Fluids 29, 046103 (2017).

[20] C. Coreixas, G. Wissocq, G. Puigt, J.-F. Boussuge, and P. Sagaut, Phys. Rev. E 96, 033306 (2017).

[21] X. He, S. Chen, and G. D. Doolen, J. Comput. Phys. 146, 282 (1998).

[22] Z. Guo, C. Zheng, B. Shi, and T. S. Zhao, Phys. Rev. E 75, 036704 (2007).

[23] Q. Li, Y. L. He, Y. Wang, and W. Q. Tao, Phys. Rev. E 76, 056705 (2007).

[24] P. Lallemand and L.-S. Luo, Int. J. Mod. Phys. B 17, 41 (2003).

[25] C. van Treeck, E. Rank, M. Krafczyk, J. Tölke, and B. Nachtwey, Comput. Fluids 35, 863 (2006).

[26] S. Bettaibi, F. Kuznik, and E. Sediki, Phys. Lett. A 378, 2429 (2014).

[27] Y. L. Feng, S. L. Guo, W. Q. Tao, and P. Sagaut, Int. J. Heat Mass Transf. 125, 1379 (2018).

[28] A. Sengissen, J.-C. Giret, C. Coreixas, and J.-F. Boussuge, in Proceedings of the 21st AIAA/CEAS Aeroacoustics Conference 
(American Institute of Aeronautics and Astronautics, Reston, VA, 2015), p. 1.

[29] W. Shao and J. Li, Arch. Acoust. 44, 215 (2019).

[30] R. Kotapati, A. Keating, S. Kandasamy, B. Duncan, R. Shock, and H. Chen, in SAE Technical Paper (The Automotive Research Association of India, Pune, India, 2009).

[31] P. L. Bhatnagar, E. P. Gross, and M. Krook, Phys. Rev. 94, 511 (1954).

[32] D. D'Humières, in Rarefied Gas Dynamics: Theory and Simulations, Vol. 159 (American Institute of Aeronautics and Astronautics, Washington, DC, 1994), pp. 450-458.

[33] D. D'Humières, I. Ginzburg, M. Krafczyk, P. Lallemand, and L.-S. Luo, Phil. Trans. R. Soc. A 360, 437 (2002).

[34] I. Ginzburg, F. Verhaeghe, and D. D'Humières, Commun. Comput. Phys. 3, 427 (2008).

[35] J. Latt and B. Chopard, Math. Comput. Simul. 72, 165 (2006).

[36] O. Malaspinas, arXiv:1505.06900 (2015).

[37] J. Jacob, O. Malaspinas, and P. Sagaut, J. Turbul. 18, 1051 (2018).

[38] M. Geier, A. Greiner, and J. G. Korvink, Phys. Rev. E 73, 066705 (2006).

[39] M. Geier, M. Schönherr, A. Pasquali, and M. Krafczyk, Comput. Math. Appl. 70, 507 (2015).

[40] M. Geier, A. Pasquali, and M. Schönherr, J. Comput. Phys. 348, 862 (2017).

[41] I. V. Karlin, A. N. Gorban, S. Succi, and V. Boffi, Phys. Rev. Lett. 81, 6 (1998).

[42] S. S. Chikatamarla, S. Ansumali, and I. V. Karlin, Phys. Rev. Lett. 97, 010201 (2006).

[43] F. Bösch, S. S. Chikatamarla, and I. V. Karlin, Phys. Rev. E 92 , 043309 (2015).

[44] R. W. Nash, H. B. Carver, M. O. Bernabeu, J. Hetherington, D. Groen, T. Krüger, and P. V. Coveney, Phys. Rev. E 89, 023303 (2014).
[45] F. Gendre, D. Ricot, G. Fritz, and P. Sagaut, Phys. Rev. E 96, 023311 (2017)

[46] M. L. Minion and D. L. Brown, J. Comput. Phys. 138, 734 (1997).

[47] H. Lamb, Hydrodynamics, 6th ed. (Cambridge University Press, Cambridge, UK, 1932).

[48] G. I. Taylor, Aero. Res. Commun. 598, 73 (1918).

[49] C. Oseen, Neuere Methoden und Ergebnisse in der Hydrodynamik (Akademie Verlag, Berlin, 1927).

[50] N. Frapolli, S. S. Chikatamarla, and I. V. Karlin, Phys. Rev. Lett. 117, 010604 (2016).

[51] B. Dorschner, F. Bösch, and I. V. Karlin, Phys. Rev. Lett. 121, 130602 (2018).

[52] M. H. Saadat, F. Bösch, and I. V. Karlin, Phys. Rev. E 99, 013306 (2019).

[53] P. J. Dellar, Phys. Rev. E 65, 036309 (2002).

[54] S. Chapman and T. Cowling, The Mathematical Theory of Nonuniform Gases: An Account of the Kinetic Theory of Viscosity, Thermal Conduction and Diffusion in Gases (Cambridge University Press, Cambridge, UK, 1970).

[55] J. C. Maxwell, Philos. Trans. R. Soc. London 157, 49 (1867).

[56] P. J. Dellar, Phys. Rev. E 64, 031203 (2001).

[57] R. Mei, L.-S. Luo, P. Lallemand, and D. d'Humières, Comput. Fluids 35, 855 (2006).

[58] K. K. Mattila, L. A. Hegele, and P. C. Philippi, Phys. Rev. E 91, 063010 (2015).

[59] G. Wissocq, P. Sagaut, and J.-F. Boussuge, J. Comput. Phys. 380, 311 (2019).

[60] R. Benzi, S. Succi, and M. Vergassola, Phys. Rep. 222, 145 (1992).

[61] O. Behrend, R. Harris, and P. B. Warren, Phys. Rev. E 50, 4586 (1994).

[62] R. Adhikari and S. Succi, Phys. Rev. E 78, 066701 (2008).

[63] P. J. Dellar, Comput. Math. Appl. 65, 129 (2013). 